\title{
THE BLOCH SPACE AND BMO ANALYTIC FUNCTIONS IN THE TUBE OVER THE SPHERICAL CONE

\author{
DAVID BEKOLLE
}

(Communicated by Irwin Kra)

\begin{abstract}
We prove that the Bloch space coincides with the space BMOA in the tube over the spherical cone of $\mathbf{R}^{3}$; this extends a well-known onedimensional result.
\end{abstract}

Introduction. Let $\Omega$ be a symmetric Siegel domain of type II contained in $\mathbf{C}^{n}$. Let $V$ denote the Lebesgue measure in $\Omega$ and $H(\Omega)$ the space of holomorphic (or analytic) functions in $\Omega$.

When $n=1$ and $\Omega=\pi^{+}=\{z \in \mathbf{C}: \operatorname{Im} z>0\}$, a Bloch function is an element $f$ of $H\left(\pi^{+}\right)$which satisfies the estimate

$$
\|f\|_{\mathscr{B}}=\sup _{z=x+i y \in \pi^{+}}\left\{y\left|f^{\prime}(z)\right|\right\}<\infty .
$$

The Bloch space $\mathscr{B}$ of $\pi^{+}$is then the quotient space of the space of Bloch functions by the subspace of constant functions.

It is well known that in $\pi^{+}$, the Bloch space $\mathscr{B}$ coincides with the quotient space BMOA of the space of BMO analytic functions by the subspace of constant functions. The definition of BMO in $\pi^{+}$is the same as that of (solid) BMO in the unit disk (cf. [6, p. 631]): in $\pi^{+}$, a locally integrable function $f$ is said to be BMO if there exists a constant $C$ such that for any disk $D$ contained in $\pi^{+}$, there is a constant $f_{D}$ such that

$$
\frac{1}{|D|} \int_{D}\left|f-f_{D}\right| d V \leq C
$$

In $\mathbf{C}^{2}$, this result can easily be extended to the cartesian product $\left(\pi^{+}\right)^{2}$ of two upper half-planes. In this case, a Bloch function is an element of $H\left[\left(\pi^{+}\right)^{2}\right]$ which satisfies the estimate

$$
\|f\|_{\mathscr{B}}=\sup _{z=\left(z_{0}, z_{1}\right)=\left(x_{0}+i y_{0}, x_{1}+i y_{1}\right) \in\left(\pi^{+}\right)^{2}}\left\{y_{0} y_{1}\left|\frac{\partial^{2}}{\partial z_{0} \partial z_{1}} f(z)\right|\right\}<\infty .
$$

The Bloch space $\mathscr{B}$ of $\left(\pi^{+}\right)^{2}$ is then the quotient space of the space of Bloch functions by the subspace

$$
\mathscr{N}=\left\{f \in H\left[\left(\pi^{+}\right)^{2}\right]: \frac{\partial^{2}}{\partial z_{0} \partial z_{1}} f(z) \equiv 0\right\}
$$

Received by the editors May 1, 1986 and, in revised form, December 4, 1986.

1980 Mathematics Subject Classification (1985 Revision). Primary 32M15, 46E99, 47B38.

Key words and phrases. Bloch space, BMO, tube over the spherical cone. 
On the other hand, a holomorphic function $f$ in $\left(\pi^{+}\right)^{2}$ is said to be BMO if there exists a constant $C$ such that for any bidisk $D$ contained in $\left(\pi^{+}\right)^{2}$, there is a function $f_{D} \in \mathscr{N}$ such that

$$
\frac{1}{|D|} \int_{D}\left|f-f_{D}\right| d V \leq C
$$

The space BMOA of $\left(\pi^{+}\right)^{2}$ is then the quotient space of the space of BMO analytic functions by the subspace $\mathscr{N}$. The referee has pointed out to the author that there is a danger of confusion between our space $\mathrm{BMO}\left[\left(\pi^{+}\right)^{2}\right]$ and the so-called space $\operatorname{BMO}\left(\mathbf{R}_{+}^{2} \times \mathbf{R}_{+}^{2}\right)$ that shows up as the dual space of the two-parameter Hardy space $H^{1}\left(\mathbf{R}_{+}^{2} \times \mathbf{R}_{+}^{2}\right)$ (cf. [4]); the two spaces, however, have nothing to do with each other.

The purpose of this paper is to extend this identity between the Bloch space and the space BMOA to the tube $\Omega$ over the spherical cone $\Gamma$ of $\mathbf{R}^{3}$ defined by

$$
\Gamma=\left\{\left(y_{0}, y_{1}, y_{2}\right) \in \mathbf{R}^{3}: y_{0} y_{1}-y_{2}^{2}>0, y_{0}>0\right\} .
$$

The Bloch space $\mathscr{B}$ of $\Omega$ is defined in [2] in terms of the differential operator

$$
\square_{z}=4 \frac{\partial^{2}}{\partial z_{0} \partial z_{1}}-\frac{\partial^{2}}{\partial z_{2}^{2}}, \quad z=\left(z_{0}, z_{1}, z_{2}\right) \in \mathbf{C}^{3} ;
$$

$\square$ is the wave operator in $\mathbf{C}^{3}$. More precisely, if $B(\varsigma, z)$ denotes the Bergman kernel of $\Omega$, a Bloch function in $\Omega$ is an element $f$ of $H(\Omega)$ which satisfies the estimate

$$
\|f\|_{\mathscr{B}}=\sup _{z \in \Omega}\left\{B^{-1 / 3}(z, z)|\square f(z)|\right\}<\infty .
$$

The Bloch space $\mathscr{B}$ of $\Omega$ is then the quotient space of the space of Bloch functions by the subspace $\mathcal{N}=\{f \in H(\Omega): \square f=0\}$.

The problem is actually to give a good definition of the space BMOA of $\Omega$; this consists of the description of a suitable family of geometric figures that will play the same role as the disks in the upper half-plane $\pi^{+}$and the bidisks in the product $\left(\pi^{+}\right)^{2}$ of two upper half-planes.

In $\S 1$, we recall some results of $[\mathbf{1}$ and 2$]$ about the Bloch space in the tube $\Omega$ over the spherical cone. According to one of these results, the Bloch space $\mathscr{B}$ of $\Omega$ can be realized as the Bergman projection of $L^{\infty}$. Let us mention that, since the Bergman kernel $B(\varsigma, z)$ of $\Omega$ is not integrable with respect to $z$, we have to state the definition of the Bergman projection $P b$ of a bounded function $b$ and we shall also recall the expression of an integral kernel which defines the operator $P$ in $L^{\infty}$. When we prove that every Bloch function $f$ is BMO, this kernel will be used in the definition of the function of $\mathcal{N}$ which will be substracted from $f$.

In $\S 2$, we define the space BMOA of $\Omega$ by describing a suitable family $\mathscr{G}$ of geometric figures: up to an affine change of coordinates, the elements of $\mathscr{G}$ are polydisks (cartesian products of three disks).

In $\S 3$, we prove the identity between the Bloch space $\mathscr{B}$ and the space BMOA of $\Omega$. Let us note that this identity can be extended with the same proof, by means of the results of $[\mathbf{3}, \mathbf{2}$ and $\mathbf{1}]$ to the following domains: the Cayley transform of the unit ball in $\mathbf{C}^{n}, n \geq 2$; the tube over the spherical cone of $\mathbf{R}^{n}, n \geq 4$; and the tube over the cone of real symmetric positive definite $m \times m$ matrices, $m \geq 3$.

The author expresses his sincere thanks to R. R. Coifman for raising this problem and to R. Rochberg for suggesting that the family should be invariant under the affine automorphisms of $\Omega$. 
1. Preliminary results about the Bloch space. In this paragraph, we recall some results proved in [1 and 2]. In the following, $\Omega$ will denote the tube $\mathbf{R}^{3}+i \Gamma \subset \mathbf{C}^{3}$ over the spherical cone $\Gamma$ of $\mathbf{R}^{3}$. The Bergman kernel $B(\zeta, z)$ of $\Omega$ has the following expression:

$$
B(\varsigma, z)=c\left[\left(\varsigma_{0}-\bar{z}_{0}\right)\left(\varsigma_{1}-\bar{z}_{1}\right)-\left(\varsigma_{2}-\bar{z}_{2}\right)^{2}\right]^{-3}
$$

where $\varsigma=\left(\varsigma_{0}, \varsigma_{1}, \varsigma_{2}\right)$ and $z=\left(z_{0}, z_{1}, z_{2}\right)$ are two points of $\Omega$.

The Bloch space $\mathscr{B}$ of $\Omega$ is defined in the introduction; equipped with the norm induced by the seminorm

$$
\|f\|_{\mathscr{B}}=\sup _{z \in \Omega}\left\{B^{-1 / 3}(z, z)|\square f(z)|\right\}
$$

defined in the space of Bloch functions, the Bloch space $\mathscr{B}$ is a Banach space.

In [2] (cf. also [1]), we extended to $\Omega$ a well-known one-dimensional result: the Bloch space can be realized as the Bergman projection of $L^{\infty}$. In fact, let us first recall that by Bergman projection, one originally means the orthogonal projection $\mathscr{P}$ of $L^{2}(d V)$ onto the Bergman space

$$
A^{2}(\Omega)=L^{2}(d V) \cap H(\Omega)
$$

defined on $L^{2}(d V)$ by

$$
\mathscr{P} \phi(\varsigma)=\int_{\Omega} B(\varsigma, z) \phi(z) d V(z), \quad \varsigma \in \Omega .
$$

We next point out that, since the Bergman kernel $B(\zeta, z)$ of $\Omega$ is not integrable with respect to $z$, we do not know how to extend to $L^{\infty}$ the domain of definition of the integral operator $\mathscr{P}$. However, we defined the Bergman projection $P$ on $L^{\infty}$ as an operator from $L^{\infty}$ into $\mathscr{B}$ satisfying the following property: when $b \in L^{\infty} \cap L^{2}$, the element $P b$ of $\mathscr{B}$ can be represented by the function $\mathscr{P} b \in A^{2}(\Omega)$.

We also determined a defining kernel for the Bergman projection of $L^{\infty}$ by subtracting from the Bergman kernel $B(\varsigma, z)$ of $\Omega$ a kernel $B_{0}(\varsigma, z)$ satisfying the two following properties:

$1^{\circ}$ The function $\varsigma \mapsto B_{0}(\varsigma, z), z \in \Omega$ fixed, is holomorphic in $\Omega$ and belongs to the zero equivalence class $\mathscr{N}$ of the Bloch space $\mathscr{B}$.

$2^{\circ}$ With respect to $z, B_{0}(\varsigma, z)$ satisfies the estimate $\left(B-B_{0}\right)(\varsigma, z) \in L^{1}(d V(z))$, $\varsigma \in \Omega$.

More explicity, a kernel $B_{0}(\varsigma, z)$ satisfying the required properties is defined by

$$
\left(B-B_{0}\right)(\varsigma, z)=\frac{\left(i-\bar{z}_{0}\right)^{5 / 2}-\left(\varsigma_{0}-\bar{z}_{0}\right)^{5 / 2}}{\left(i-\bar{z}_{0}\right)^{5 / 2}}\left\{B(\varsigma, z)-B\left(\left(\varsigma_{0}, i, 0\right), z\right)\right\},
$$

i.e. for any $b \in L^{\infty}$, the function

$$
g(\varsigma)=\int_{\Omega}\left(B-B_{0}\right)(\varsigma, z) b(z) d V(z)
$$

is an element of the equivalence class $P b$ of $\mathscr{B}$.

The following theorem is proved in [2]. 
THEOREM 1.1. The Bergman projection $P$, which assigns to a bounded function $b$ the element $\mathrm{Pb}$ of $\mathscr{B}$ represented by the Bloch function $g$ defined by (2), is a continuous operator from $L^{\infty}$ onto the Bloch space $\mathscr{B}$ of $\Omega$. Furthermore, the projection $P$ possesses a continuous right inverse $R: \mathscr{B} \rightarrow L^{\infty}$.

Let us mention the following lemma, which was used in the proof of the preceding theorem.

LEMMA 1.1. Let $K$ be a compact subset of $\Omega$. There exist a constant $C=C(K)$ and a positive function $M \in L^{1}(d V)$ such that for any $\varsigma \in K$ and any $z \in \Omega$, the following inequality holds:

$$
\left|\left(B-B_{0}\right)(\varsigma, z)\right| \leq C M(z) .
$$

2. The space BMOA. In the introduction, we reduced the definition of the space BMOA to the description of a suitable family $\mathscr{G}$ of geometric figures.

Let us then describe such a family $\mathscr{G}$. We first describe a subfamily $\mathscr{G}_{0}$ of $\mathscr{G}_{;} \mathscr{G}_{0}$ will consist of all polydisks centered at $e=(i, i, 0)$, whose multiradius $\left(R_{0}, R_{1}, R_{2}\right)$ satisfies $0<R_{j} \leq 1 / 4$ for $j=0,1,2$. Notice that those polydisks are all contained in $\Omega$.

Now, let $\operatorname{Aff}(\Omega)$ denote the simply transitive group of affine automorphisms of $\Omega$ defined in the first paragraph of [7]; the whole family $\mathscr{G}$ is defined by

$$
\mathscr{G}=\bigcup_{\phi \in \operatorname{Aff}(\Omega)} \phi\left(\mathscr{G}_{0}\right)
$$

Let us describe more precisely the figures of $\mathscr{G}$. We shall represent the domain $\Omega$ by the space of symmetric $2 \times 2$ matrices in the following way: a point $z=\left(z_{0}, z_{1}, z_{2}\right)$ of $\Omega$ will take the form

$$
z=\left(\begin{array}{ll}
z_{0} & z_{2} \\
z_{2} & z_{1}
\end{array}\right)
$$

Now, let

$$
\varsigma=s+i t=\left(\begin{array}{ll}
s_{0}+i t_{0} & s_{2}+i t_{2} \\
s_{2}+i t_{2} & s_{1}+i t_{1}
\end{array}\right), \quad s \in \mathbf{R}^{3}, t \in \Gamma
$$

be a point of $\Omega$; we shall use the following notation, introduced in [7]:

$$
\chi_{1}(t)=\frac{t_{0} t_{1}-t_{2}^{2}}{t_{1}} \quad \text { and } \quad \chi_{2}(t)=t_{1}
$$

Let $\phi$ be the element of $\operatorname{Aff}(\Omega)$ that assigns $\varsigma$ to $e ; \phi$ is defined by $\phi=\phi_{3} \circ \phi_{2} \circ \phi_{1}$, where the elements $\phi_{1}, \phi_{2}, \phi_{3}$ of $\operatorname{Aff}(\Omega)$ are defined as follows for $z \in \Omega$;

$\phi_{1}(z)=g_{1} z g_{1}$, where $g_{1}$ denotes the diagonal matrix

$$
g_{1}=\left(\begin{array}{cc}
\chi_{1}(t)^{1 / 2} & 0 \\
0 & \chi_{2}(t)^{1 / 2}
\end{array}\right)
$$

$\phi_{2}(z)=g_{2} z g_{2}^{\prime}$, where $g_{2}$ denotes the upper triangular matrix

$$
g_{2}=\left(\begin{array}{cc}
1 & \frac{t_{2}}{t_{1}} \\
0 & 1
\end{array}\right)
$$

and $g_{2}^{\prime}$ is the transpose matrix of $g_{2}$;

$$
\phi_{3}(z)=z+s=\left(\begin{array}{ll}
z_{0}+s_{0} & z_{2}+s_{2} \\
z_{2}+s_{2} & z_{1}+s_{1}
\end{array}\right) .
$$


Let $Q$ be a figure of $\mathscr{G}, Q=\phi(D)$, where $D$ is an element of $\mathscr{G}_{0}$ whose multiradius is denoted by $\left(R_{0}, R_{1}, R_{2}\right)$. A point $z=\left(z_{0}, z_{1}, z_{2}\right)$ belongs to $Q$ if

$$
\left\{\begin{array}{l}
\left|z_{1}-\varsigma_{1}\right|<\chi_{2}(t) R_{1}, \\
\left|z_{2}-\varsigma_{2}-\frac{t_{2}}{\chi_{2}(t)}\left(z_{1}-\varsigma_{1}\right)\right|<\left[\chi_{1}(t) \chi_{2}(t)\right]^{1 / 2} R_{2}, \\
\left|z_{0}-\varsigma_{0}-\frac{2 t_{2}}{\chi_{2}(t)}\left(z_{2}-\varsigma_{2}\right)+\left(\frac{t_{2}}{\chi_{2}(t)}\right)^{2}\left(z_{1}-\varsigma_{1}\right)\right|<\chi_{1}(t) R_{0} .
\end{array}\right.
$$

Now, by means of the affine change of coordinates defined by

$$
\left\{\begin{array}{l}
z_{1}^{\prime}=z_{1}, \\
z_{2}^{\prime}=z_{2}-\frac{t_{2}}{\chi_{2}(t)}\left(z_{1}-\varsigma_{1}\right), \\
z_{0}^{\prime}=z_{0}-\frac{2 t_{2}}{\chi_{2}(t)}\left(z_{2}-\varsigma_{2}\right)+\left(\frac{t_{2}}{\chi_{2}(t)}\right)^{2}\left(z_{1}-\varsigma_{1}\right),
\end{array}\right.
$$

we obtain that $Q$ is the polydisk centered at $\varsigma=\left(\varsigma_{0}, \varsigma_{1}, \varsigma_{2}\right)$, defined by

$$
\begin{aligned}
Q=\left\{\left(z_{0}^{\prime}, z_{1}^{\prime}, z_{2}^{\prime}\right) \in \mathbf{C}^{3}:\left|z_{1}^{\prime}-\varsigma_{1}\right|\right. & <\chi_{2}(t) R_{1}, \\
\left|z_{2}^{\prime}-\varsigma_{2}\right| & \left.<\left[\chi_{1}(t) \chi_{2}(t)\right]^{1 / 2} R_{2},\left|z_{0}^{\prime}-\varsigma_{0}\right|<\chi_{1}(t) R_{0}\right\} .
\end{aligned}
$$

Let us next define the space BMOA of $\Omega$. A function $f \in H(\Omega)$ is said to be BMO if there exists a constant $C$ such that for any $Q \in \mathscr{G}$, there is a function $f_{Q} \in \mathcal{N}$, where $\mathcal{N}=\{f \in H(\Omega): \square f=0\}$, such that

$$
\frac{1}{|Q|} \int_{Q}\left|f-f_{Q}\right| d V \leq C
$$

Let $\|f\|_{\mathrm{BMOA}}$ denote the smallest constant for which this property holds. As a definition, the space BMOA of $\Omega$ is the quotient space of the BMO analytic functions by the subspace $\mathscr{N}$; equipped with the norm induced by \|\|$_{\text {BMOA }}$, the space BMOA is a Banach space.

3. The identity between the Bloch space $\mathscr{B}$ and the space BMOA. We shall now prove the following theorem.

THEOREM 3.1. In the tube $\Omega$ over the spherical cone $\Gamma$ of $\mathbf{R}^{3}$, the Bloch space $\mathscr{B}$ coincides with the space $\mathrm{BMOA}$ and the norms induced on $\mathscr{B}$ by \|\|$_{\mathscr{B}}$ and on BMOA by \|\|$_{\mathrm{BMOA}}$ are equivalent.

ProOF. We first show that there exists a constant $C$ such that any Bloch function $f$ satisfies $\|f\|_{\mathrm{BMOA}} \leq C\|f\|_{\mathscr{B}}$. This will immediately yield the inclusion of $\mathscr{B}$ in BMOA.

Let then $f$ be a Bloch function; in the following, $B_{0}$ denotes the kernel defined in [1]. In view of Theorem 1.2, there exists a bounded function $b$ such that the following equality holds, modulo an element of $\mathcal{N}$ :

$$
f(\varsigma)=\int_{\Omega}\left(B-B_{0}\right)(\varsigma, z) b(z) d V(z)
$$

i.e. $f$ belongs to the same equivalence class of $\mathscr{B}$ as the function in the right-hand side of (3). 
We must prove that there exists a constant $C$ such that for any Bloch function $f$ and any polydisk $Q \in \mathscr{G}$, there is a function $f_{Q} \in \mathcal{N}$ such that

$$
\frac{1}{|Q|} \int_{Q}\left|f-f_{Q}\right| d V \leq C\|f\|_{\mathscr{B}} .
$$

Let $c$ denote the center of $Q$ and let $\phi$ be the element of $\operatorname{Aff}(\Omega)$ that assigns $c$ to $e$. Define the function $f_{Q}$ by

$$
\left(f-f_{Q}\right)(\varsigma)=\int_{\Omega}\left(B-B_{0}\right)(\phi(\varsigma), \phi(z)) b(z)|J \phi|^{2} d V(z)
$$

where $J \phi$ denotes the jacobian of $\phi$.

Let us verify that the so-defined function $f_{Q}$ belongs to $\mathscr{N}$; in view of Lemma 1.1, (3) and the relation $B(\phi(\varsigma), \phi(z))|J \phi|^{2}=B(\varsigma, z)$, it suffices to check that

$$
\square_{\varsigma}\left\{B_{0}(\phi(\varsigma), \phi(z))|J \phi|^{2}-B_{0}(\varsigma, z)\right\} \equiv 0,
$$

and since $\square_{\varsigma} B_{0}(\zeta, z) \equiv 0$ and $J \phi$ is independent of $\varsigma$, this is equivalent to

$$
\square_{\varsigma} B_{0}(\phi(\varsigma), \phi(z)) \equiv 0 \text {. }
$$

This verification then follows from the following lemma, whose proof is elementary.

LEMMA 3.1. For any $f \in H(\Omega)$ and any $\phi \in \operatorname{Aff}(\Omega)$, the following equality holds.

$$
\square(f \circ \phi)=|J \phi|^{2 / 3} \cdot(\square f) \circ \phi .
$$

Let us next prove that the function $f_{Q}$ defined in (5) gives rise to inequality (4). In view of (5) and the Fubini theorem, we get

$$
\frac{1}{|Q|} \int_{Q}\left|\left(f-f_{Q}\right)(\varsigma)\right| d V(\varsigma) \leq \int_{\Omega} N(z) b(z) d V(z) \leq\|b\|_{\infty} \int_{\Omega} N(z) d V(z),
$$

where

$$
N(z)=\frac{1}{|Q|} \int_{Q}\left|\left(B-B_{0}\right)(\phi(\varsigma), \phi(z))\right||J \phi|^{2} d V(\varsigma)
$$

Now, in view of Theorem 1.1, if $R$ denotes the continuous right inverse of $P: L^{\infty} \rightarrow \mathscr{B}$, we can take $b=R f$ and this yields the estimate $\|b\|_{\infty} \leq C\|f\|_{\mathscr{B}}$.

It then suffices to show that there exists a constant $C^{\prime}$ such that for any $Q \in \mathscr{G}$, the function $N$ defined in (6) satisfies the estimate

$$
\int_{\Omega} N(z) d V(z) \leq C^{\prime}
$$

In order to prove (7), we apply to (6) the change of variables $\varsigma^{\prime}=\phi(\varsigma)$ and $z^{\prime}=$ $\phi(z)$ and we let $D$ denote the polydisk $D=\phi^{-1}(Q)$ centered at $e$ ( $D$ belongs to the subfamily $\mathscr{G}_{0}$ of $\mathscr{G}$ ); since $|Q|=|J \phi|^{-2}|D|$, it follows from the Fubini theorem that the left-hand side of $(7)$ is equal to $(1 /|D|) \int_{D}\left\{\int_{\Omega}\left|\left(B-B_{0}\right)\left(\varsigma^{\prime}, z^{\prime}\right)\right| d V\left(z^{\prime}\right)\right\} d V\left(\varsigma^{\prime}\right)$.

Applying Lemma 1.1 to the compact subset

$$
\bar{D}_{0}=\left\{\left(z_{0}, z_{1}, z_{2}\right) \in \mathbf{C}^{3}:\left|z_{0}-i\right| \leq 1 / 4,\left|z_{1}-i\right| \leq 1 / 4,\left|z_{2}\right| \leq 1 / 4\right\}
$$

immediately yields inequality (7). 
We now prove the converse implication, i.e. there exists a constant $C$ such that any $\mathrm{BMO}$ analytic function $f$ satisfies the estimate $\|f\|_{\mathscr{B}} \leq C\|f\|_{\mathrm{BMOA}}$.

The method of proof is the same as that of R. R. Coifman, R. Rochberg and G. Weiss [6] for the same property in the unit disk of the complex plane.

We must show that there exists a constant $C$ such that for any BMO analytic function $f$ and any $s$ in $\Omega$, the following inquality holds:

$$
B^{-1 / 3}(\varsigma, \varsigma)|\square f(\varsigma)| \leq C\|f\|_{\mathrm{BMOA}}
$$

Let us first prove inequality (8) for any $\zeta$ in the polydisk $D$ centered at $e$, whose multiradius is $(1 / 5,1 / 5,1 / 5)$. We let $D_{0}$ denote the polydisk centered at $e$, whose multiradius is $(1 / 4,1 / 4,1 / 4)$ and we let $f_{0}$ denote an element of $\mathscr{N}$ such that

$$
\frac{1}{\left|D_{0}\right|} \int_{D_{0}}\left|f-f_{0}\right| d V \leq\|f\|_{\mathrm{BMOA}}
$$

We use the following lemma.

LEMMA 3.2. If $g \in H(\Omega)$ satisfies $\left(1 /\left|D_{0}\right|\right) \int_{D_{0}}|g| d V \leq C<\infty$, then for any $\varsigma$ in $D$, the inequality $|\square g(\varsigma)| \leq 135 C$ holds.

PROOF OF LEMMA 3.2. In view of Cauchy's formula for polydisks, the following equality holds for any $\zeta$ in $D$ :

$$
\begin{aligned}
\frac{\partial^{2}}{\partial \zeta_{0} \partial \zeta_{1}} g(\varsigma)=\left(\frac{400}{9 \pi i}\right)^{3} \int_{r_{2}}^{3} & =1 / 5 \\
& \times\left\{\int_{r_{1}=1 / 5}^{1 / 4} \frac{g(z) d z_{0} \wedge d z_{1} \wedge d z_{2}}{\left(z_{0}-\varsigma\right)^{2}\left(z_{1}-\varsigma_{1}\right)^{2}\left(z_{2}-\varsigma_{2}\right)}\right\} r_{0} d r_{0} r_{1} d r_{1} r_{2} d r_{2}
\end{aligned}
$$

where

$$
\partial D_{r}=\left\{\left(z_{0}, z_{1}, z_{2}\right) \in \mathbf{C}^{3}:\left|z_{0}-i\right|=r_{0},\left|z_{1}-i\right|=r_{1},\left|z_{2}\right|=r_{2}\right\} .
$$

We then easily obtain that

$$
\left|\frac{\partial^{2}}{\partial \zeta_{0} \partial \zeta_{1}} g(\varsigma)\right| \leq\left(\frac{25}{9}\right)^{3} \frac{1}{\left|D_{0}\right|} \int_{D_{0}}|g| d V
$$

and the same estimate also holds for $\left|\left(\partial^{2} / \partial \varsigma_{2}^{2}\right) g(\varsigma)\right|$ : the conclusion immediately follows from the hypothesis.

Now, in view of Lemma 3.2 and of the equality $\square f_{0}=0$, inequality (8) when $\varsigma$ lies in $D$ is a consequence of the following lemma, whose proof is easy.

LEMMA 3.3. There exist two positive constants $C_{1}$ and $C_{2}$ such that for any $\varsigma$ in $D, C_{1} \leq B(\varsigma, \varsigma) \leq C_{2}$.

Let us next prove inequality (8) when $\zeta$ is any point in $\Omega$. We let $D$ again denote the polydisk centered at $e$, whose multiradius is $(1 / 5,1 / 5,1 / 5)$; since the family $\{\phi(D): \phi \in \operatorname{Aff}(\Omega)\}$ is a covering of $\Omega$, it is enough to prove that for any $\phi \in \operatorname{Aff}(\Omega)$, inequality (8) holds for any $\varsigma$ in $\phi(D)$.

Let $Q=\phi(D)$ and $Q_{0}=\phi\left(D_{0}\right)$, where $D_{0}$ again denotes the polydisk centered at $e$ whose multiradius is $(1 / 4,1 / 4,1 / 4)$. Let $f_{\phi}$ be an element of $\mathscr{N}$ such that

$$
\frac{1}{\left|Q_{0}\right|} \int_{Q_{0}}\left|\left(f-f_{\phi}\right)(z)\right| d V(z) \leq\|f\|_{\mathrm{BMOA}} \text {. }
$$


Applying the change of variable $z=\phi\left(z^{\prime}\right)$ to the left-hand side of (9) yields

$$
\frac{1}{\left|D_{0}\right|} \int_{D_{0}}\left|\left(f-f_{\phi}\right) \circ \phi\left(z^{\prime}\right)\right| d V\left(z^{\prime}\right) \leq\|f\|_{\mathrm{BMOA}} .
$$

Now, in view of Lemma 3.3, we get for any $\varsigma^{\prime}$ in $D$ :

$$
\left|\square_{\varsigma^{\prime}}\left[\left(f-f_{\phi}\right) \circ \phi\right]\left(\varsigma^{\prime}\right)\right| \leq 135\|f\|_{\mathrm{BMOA}} .
$$

We next apply the change of variable $s=\phi\left(s^{\prime}\right)$ to the left-hand side of this inequality; in view of Lemma 3.1 and of the equality $\square f_{\phi}=0$, this yields

$$
\left|\square_{\varsigma} f(\varsigma)\right||J \phi|^{2 / 3} \leq 135\|f\|_{\text {BMOA }} \text {. }
$$

The following lemma then completes the proof of the theorem.

LEMMA 3.4. D denotes the polydisk centered at $e$, whose multiradius is $(1 / 5$, $1 / 51 / 5)$. There exist two constants $C_{1}$ and $C_{2}$ such that for any $\phi \in \operatorname{Aff}(\Omega)$ and any $\varsigma \in \phi(D)$,

$$
C_{1}|J \phi|^{-2} \leq B(\varsigma, \varsigma) \leq C_{2}|J \phi|^{-2} .
$$

PROOF OF LEMMA 3.4. This lemma is a straightforward consequence of Lemma 3.2 and of the relation $B(\varsigma, \varsigma)=B\left(\varsigma^{\prime}, \varsigma^{\prime}\right)|J \phi|^{-2}$, where $\varsigma=\phi\left(\varsigma^{\prime}\right)$.

\section{REFERENCES}

1. D. Békollé, Espaces de fonctions holomorphes dans des domaines de $\mathbf{C}^{n}$, Thèse d'Etat, Orléans, 1984.

2. _ـ Le dual de l'espace des fonctions holomorphes intégrables dans des domaines de Siegel, Ann. Inst. Fourier (Grenoble) 33 (1984), 125-154.

3. __ Le dual de la classe de Bergman $A^{1}$ dans le transformé de Cayley de la boule unité de $\mathbf{C}^{n}$, C. R. Acad. Sci. Paris Sér I 296 (1983), 377-380.

4. S.-Y. A. Chang and R. Fefferman, Some recent developments in Fourier analysis and $H^{p}$ theory on product domains, Bull. Amer. Math. Soc. (N.S.) 12 (1985), 1-43.

5. R. R. Coifman and R. Rochberg, Representation theorems for holomorphic and harmonic functions in $L^{p}$, Astérisque 77 (1980), 11-66.

6. R. R. Coifman, R. Rochberg and G. Weiss, Factorization theorems for Hardy spaces in several variables, Ann. of Math. (2) 103 (1976), 611-635.

7. S. G. Gindikin, Analysis in homogeneous domains, Russian Math. Surveys 19 (1964), no. 4, 1-89.

DÉPARTEMENT de Mathématiques, UniVersité de YaOUndé, BP. 812, YaOUNDÉ, CAMEROUN 